\title{
ORDERED INVOLUTIVE OPERATOR SPACES
}

\author{
DAVID P. BLECHER, KAY KIRKPATRICK, MATTHEW NEAL, AND WEND WERNER
}

\begin{abstract}
This is a companion to recent papers of the authors; here we construct the 'noncommutative Shilov boundary' of a (possibly nonunital) selfadjoint ordered space of Hilbert space operators. The morphisms in the universal property of the boundary preserve order. As an application, we consider 'maximal' and 'minimal' unitizations of such ordered operator spaces.
\end{abstract}

\section{INTRODUCTION}

An operator space is a closed linear space of bounded operators between Hilbert spaces, or, equivalently, a subspace of a $\mathrm{C}^{*}$-algebra. Although ordered operator spaces containing the identity operator are well understood and important (these are known as operator systems [7, 14]), nonunital ordered operator spaces have barely been studied at all. This is despite the fact that they occur very naturally; for example, consider the linear span of three generic positive matrices in the $5 \times 5$ matrices $M_{5}$. Indeed, the only theory addressing such spaces which we are aware of is contained in [15, 16, 17, 6, 5] and a series of papers by Karn (see e.g. 10, 11] and references therein). In view of the importance of the notion of operator positivity, we offer, in this companion paper to [6, 5], some results on this topic. In particular, we construct the 'noncommutative Shilov boundary' of such a space $X$, and use this to construct a 'maximal unitization' of $X$. At the end of the paper we will illustrate these two main concepts in the very simple case of spaces of finite matrices.

Our main tool is the 'noncommutative Shilov boundary'. We remark that this tool was used from the beginning in the case of unital operator systems [1, and the commutative variant is fundamental in the theory of classical function spaces. In 2001, as a 'Research Experience for Undergraduates' project, the second author considered the possibility of a noncommutative Shilov boundary for 'selfadjoint operator spaces' [12. It is well known (see e.g. 9, 4]) that the noncommutative analogue of the Shilov boundary of an operator space should be a ternary ring of operators, or TRO for short. A TRO is a closed subspace of a $\mathrm{C}^{*}$-algebra which is closed under products of the form $x y^{*} z$. If $X$ is selfadjoint then its enveloping TRO will also be selfadjoint, that is, it is a '*-TRO'. In [6] the first and last author developed the theory of $*$-TROs, and the ways in which they can be ordered. If $X$ is an ordered operator space, then one might hope that the 'arrows' in the universal diagram/property for the noncommutative Shilov boundary may be chosen to be

1991 Mathematics Subject Classification. Primary 47L07, 47L05; Secondary 46B40, 46L07, 46L08, 47B60, 47B65.

To appear, Positivity.

*Blecher was partially supported by grant DMS 0400731 from the National Science Foundation. Kirkpatrick was partially supported by an NSF REU grant. Neal was supported by Denison University. Werner was supported by the SFB 487 Geometrische Strukturen in der Mathematik, at the Westfälische Wilhelms-Universität, supported by the Deutsche Forschungsgemeinschaft. 
(completely) positive. This was done in [5] in a general setting. In the present paper we give the technical details of how to adapt these results to the case of selfadjoint operator spaces. For example, the proof of the just mentioned universal property is necessarily quite different in our setting here. As an application, in the last part of the paper, we discuss 'maximal' and 'minimal' unitizations of nonunital ordered operator spaces.

Any unexplained notation can be found in 6, 5, 4, A map $T: X \rightarrow Y$ between vector spaces which have involutions is called $*$-linear or selfadjoint, if it is linear and $i\left(x^{*}\right)=i(x)^{*}$ for all $x \in X$. A selfadjoint operator space is an operator space $X$ with an involution $*: X \rightarrow X$, such that there exists a complete isometry $i: X \rightarrow B(H)$ which is $*$-linear. Alternatively, a selfadjoint operator space is an operator space $X$ with an involution $*: X \rightarrow X$, such that

$$
\left\|\left[x_{j i}^{*}\right]\right\|=\left\|\left[x_{i j}\right]\right\|, \quad n \in \mathbb{N},\left[x_{i j}\right] \in M_{n}(X) .
$$

(To see the nontrivial direction of this equivalence, suppose that $X$ is a concrete (not necessarily selfadjoint) subspace of $B(H)$, and that $\tau: X \rightarrow X$ is an involution satisfying the last centered equation. Then the map

$$
X \longrightarrow M_{2}(B(H)): x \mapsto\left[\begin{array}{cc}
0 & x \\
\tau(x)^{*} & 0
\end{array}\right],
$$

is a $*$-linear complete isometry of $X$ onto a selfadjoint subspace of a $\mathrm{C}^{*}$-algebra.)

By an ordered operator space, we mean a selfadjoint operator space $X$ with a specified cone $\mathfrak{c}_{n} \subset M_{n}(X)$ for each $n \in \mathbb{N}$, such that there exists a completely isometric $*$-linear map $T: X \rightarrow B(H)$ such that $T$ is completely positive in the sense that $T_{n}\left(\mathfrak{c}_{n}\right) \subset M_{n}(B(H))_{+}$, for all $n \in \mathbb{N}$. We warn the reader that this notation is nonstandard, in other papers $T$ is usually also required to be a complete order embedding, that is $T^{-1}$ is also completely positive on $\operatorname{Ran}(T)$. An ordered operator space is often written as a pair $\left(X,\left(\mathfrak{c}_{n}\right)\right)$. We sometimes write $\mathfrak{c}_{n}$ as $M_{n}(X)_{+}$, and write $x \geq 0$ for $x$ in this cone. There is another important variant of the theory, where we have only one cone $\mathfrak{c} \subset X$, and $T$ above is positive. However since this variant works out almost identically, we will not explicitly mention it.

We end this introduction with the following remark which we will refer to later. One initial motivation to study nonunital ordered operator spaces comes from the fact that the dual of a $\mathrm{C}^{*}$-algebra has a very nice positive cone (namely, the positive linear functionals), and is an operator space [4, Section 1.4]. This leads one to hope that it may be treated as an ordered operator space. However the situation here is certainly more difficult than one might first think:

Proposition 1.1. If $A^{\prime}$ is the dual Banach space of a nontrivial $C^{*}$-algebra $A$, with its usual cone, then there exists no isometric positive map from $A^{\prime}$ into another $C^{*}$ algebra. Thus $A^{\prime}$ is not an ordered operator space.

Proof. First suppose that $A=\ell_{2}^{\infty}$. In this case this result was mentioned in [5] as a consequence of some rather intricate facts. However there is a simple direct proof. Suppose that $u: \ell_{2}^{1} \rightarrow B(H)$ is an isometric positive map. If $u\left(e_{i}\right)=T_{i}$, then $T_{i}$ are positive contractions. Since $T_{1}-T_{2}$ is selfadjoint, a well known formula gives

$$
\left\|T_{1}-T_{2}\right\|=\sup \left\{\left|\left\langle\left(T_{1}-T_{2}\right) \zeta, \zeta\right\rangle\right|: \zeta \in \operatorname{Ball}(H)\right\} \leq 1 .
$$

This contradicts the fact that $2=\|(1,-1)\|_{\ell_{2}^{1}}=\left\|T_{1}-T_{2}\right\|$.

We finish the proof by proving the more general fact that if $M$ is any von Neumann algebra of dimension $>1$, then there exists no positive isometry $T$ from 
the predual $M_{*}$ into another $\mathrm{C}^{*}$-algebra $B$. For if there did exist such $T$, then $T^{\prime \prime}: M^{\prime} \rightarrow B^{\prime \prime}$ would be a positive isometry into a $\mathrm{C}^{*}$-algebra. Let $p$ be a nontrivial projection in $M$, and let $N=\operatorname{Span}\left\{p, p^{\perp}\right\}$, the injective 2 dimensional $\mathrm{C}^{*}$-algebra. By injectivity, there exists a completely positive unital projection $P: M \rightarrow N$. Then $P^{\prime}: N^{\prime} \rightarrow M^{\prime}$ is a positive isometry. Thus $T^{\prime \prime} \circ P^{\prime}$ induces a positive isometry from $\ell_{2}^{1}$ into a $\mathrm{C}^{*}$-algebra, contradicting the previous paragraph.

\section{The Selfadjoint and the ordered nOncommutative Shilov Boundary}

The natural morphisms between TROs are the ternary morphisms, that is the maps satisfying $T\left(x y^{*} z\right)=T(x) T(y)^{*} T(z)$. As mentioned in the introduction of $[\underline{6}$, the basic facts about TROs and their ternary morphisms have selfadjoint variants valid for $*$-TROs and $*$-linear ternary morphisms. We will use the term 'ternary *-morphism' for the latter. Similarly, as we shall see next, there is a selfadjoint variant of the noncommutative Shilov boundary of an operator space. That is, Hamana's theory of ternary envelopes (see 9, 4]) easily restricts to the context of selfadjoint subspaces of $\mathrm{C}^{*}$-algebras.

By a ternary $*$-extension of a selfadjoint operator space $X$, we mean a pair $(Z, i)$ consisting of a $*$-TRO $Z$, and a $*$-linear complete isometry $i: X \rightarrow Z$ such that 'odd polynomials' with variables taken from $i(X)$ are dense in $Z$. Equivalently, there is no nontrivial subTRO of $Z$ containing $i(X)$. We define a ternary $*$-envelope of $X$ to be any ternary $*$-extension $(Y, i)$ with the universal property of the next theorem. This could also be called the 'selfadjoint noncommutative Shilov boundary'.

Theorem 2.1. If $X$ is a selfadjoint operator space, then there exists a ternary *-extension $(Y, j)$ of $X$ with the following universal property: Given any ternary *-extension $(Z, i)$ of $X$ there exists a (necessarily unique and surjective) selfadjoint ternary morphism $\pi: Z \rightarrow Y$, such that $\pi \circ i=j$.

Proof. Let $(Z, i)$ be a ternary $*$-extension of $X$. By the basic theory of the ternary envelope (see 9] or 8.3.10 in [4]), there exists a subbimodule $N$ of $Z$ such that $Z / N$ is a copy of the ternary envelope of $X$. For any operator space $X$, the adjoint space $X^{*}=\left\{x^{*}: x \in X\right\}$ is a well defined operator space, independent of the representation of $X[4,1.2 .25]$. We have $(X / N)^{*} \cong X^{*} / N^{*}$ completely isometrically, using [4, 1.2.15], for example. Since the canonical map $j: X \rightarrow Z / N$ is completely isometric, so is the canonical map $X=X^{*} \rightarrow(Z / N)^{*} \cong Z^{*} / N^{*}=Z / N^{*}$. However $N$ is the largest subbimodule $W$ of $Z$ such that the canonical map $X \rightarrow Z / W$ is completely isometric (see [9] or [2, Theorem A11]). Thus $N^{*} \subset N$, so that $N=N^{*}$. Hence the ternary envelope $Y=Z / N$ of $X$ is a $*$-TRO, by observations in the introduction to [6], and hence is a ternary $*$-extension of $X$. If $(W, k)$ is any ternary *-extension of $X$, then $W$ is a ternary extension of $X$, and by the universal property of the envelope there exists a (necessarily unique and surjective) ternary morphism $\pi: W \rightarrow Y$ with $\pi \circ k=j$. It is easy algebra to check that $\pi$ is $*$-linear.

It is now routine to extend the basic properties of ternary envelopes (see e.g. 9, 2. or [4, 8.3.10 and 8.3.12]) to ternary *-envelopes. We omit the details. Indeed, part of the proof above shows, using routine arguments, that any ternary $*$-envelope of $X$ is a ternary envelope of $X$.

Suppose next that $X$ is an ordered operator space, with matrix cones $\left(\mathfrak{c}_{n}\right)$. We consider an ordered version of the ternary envelope or noncommutative Shilov boundary. That is, we seek an ordered version of the universal property/diagram 
in Theorem 2.1. Except under extra hypotheses (for example, if the positive cone of $X$ densely spans $X$ ), it is easy to show that the embeddings $i: X \rightarrow Z$ occurring in the universal property/diagram in the ordered case cannot be allowed in general to be arbitrary complete order embeddings, or even arbitrary completely positive complete isometries. We will usually need to limit the size of the cone of $Z$. Fortunately there is an appropriate bound for this cone, and this bound depends on the given cone of $X$.

More specifically, we assign a canonical cone $\mathfrak{d}$ to the ternary $*$-envelope $(\mathcal{T}(X), j)$, namely the intersection of all natural cones (in the sense of [6] ) containing $\left(j\left(\mathfrak{c}_{n}\right)\right)$. To see that there exists at least one such cone, note that if $i: X \rightarrow B$ is a completely positive complete isometry into a $\mathrm{C}^{*}$-algebra, and if $W$ is the $*$-TRO generated by $i(X)$, then by Theorem 2.1 there is a ternary $*$-morphism $\theta: W \rightarrow \mathcal{T}(X)$ with $\theta \circ i=j$. Thus $\mathcal{T}(X)$ is ternary $*$-isomorphic to a quotient of $W$. By [6, Lemma $5.2]$, this quotient of $W$ has a natural cone containing the image of $i(\mathfrak{c})$.

As mentioned in [6, Section 4], the intersection of natural cones (in the sense of [6]) is natural. Hence $\mathfrak{d}$ is a natural cone on $\mathcal{T}(X)$ containing $j(\mathfrak{c})$. We call $\mathcal{T}(X)$ equipped with this cone the ordered ternary envelope, and write it as $\mathcal{T}^{\circ}(X)$. This is the 'ordered noncommutative Shilov boundary'. We remark that up to the obvious notion of equivalence, the ordered ternary envelope is well defined independently of any particular ternary $*$-envelope of $X$. This follows for example from the universal property below.

The following result is reminiscent of [5, Theorem 5.3], but seems to require a completely different proof. (Note that the proof in the setting considered in [5] needs to use the notion of 'range tripotents'. Since these may not be 'central' in the sense of [6], they will not work for us here.

Theorem 2.2. Suppose that $(X, \mathfrak{c})$ is an ordered operator space. Suppose that $\iota$ is $a *$-linear completely positive complete isometry from $X$ into $a *$-TRO $Z$ such that $\iota(X)$ generates $Z$ as a $*$-TRO. Let $\mathfrak{e}$ denote the intersection of all natural cones of $Z$ (in the sense of [6]) containing $\iota(\mathfrak{c})$. Let $\mathfrak{d}$ be the natural cone defining the ordered ternary $*$-envelope $\mathcal{T}^{\circ}(X)$. Then the canonical ternary $*$-morphism $\theta: Z \rightarrow \mathcal{T}^{o}(X)$ from Theorem 2.1, takes $\mathfrak{e}$ onto $\mathfrak{d}$.

Proof. This requires basic results and notation from [6]. Let $u$ be the tripotent in $\mathcal{T}(X)^{\prime \prime}$ associated (by the correspondence from [6, Theorem 4.16]) with the natural cone $\mathfrak{d}$ in $\mathcal{T}(X)$. We write $J(u)$ for the 'Pierce 2-space' of $u$ in $\mathcal{T}(X)^{\prime \prime}$, namely the subset of elements satisfying $y=u y u$. The inverse image under the ternary $*-$ morphism $\theta^{\prime \prime}$ of $J(u)$ is a weak*-closed ternary $*$-ideal $I$ of $Z^{\prime \prime}$. Thus, $Z^{\prime \prime}=I \oplus^{\infty} J$ for some ternary $*$-ideal $J$ (see e.g. [6, Lemma $3.4(1)]$ ). Now let $z$ be the tripotent associated with the natural cone $\mathfrak{e}$. Then $z=z_{1}+z_{2}$ for orthogonal tripotents $z_{1} \in I$ and $z_{2} \in J$. Since $z$ is central and selfadjoint, it follows easily that $z_{1}$ is central and selfadjoint. Because $\theta^{\prime \prime}$ takes open central tripotents to open central tripotents (by a variant of [5. Proposition 3.5]), and hence natural cones to natural cones, $\theta^{\prime \prime}(z) \geq u$. Since $z_{2} I=0$, it is also clear that $\theta^{\prime \prime}\left(z_{2}\right)$ is orthogonal to $u$. Thus, $\theta^{\prime \prime}\left(z_{1}\right) \geq u$. But $\theta^{\prime \prime}\left(z_{1}\right) \in J(u)$, and so $\theta^{\prime \prime}\left(z_{1}\right)=u \theta^{\prime \prime}\left(z_{1}\right) u=u$. It follows that $\theta^{\prime \prime}\left(J\left(z_{1}\right)\right) \subset J(u)$, where $J\left(z_{1}\right)$ is the Pierce 2-space of $z$ in $Z^{\prime \prime}$.

Recall that $\iota(\mathfrak{c})$ lies in $J(z)_{+}$, the positive part of the Pierce 2-space of $z$. Thus, for any $x \in \iota(\mathfrak{c})$, we have $x=z^{2} x=z_{1}^{2} x+z_{2}^{2} x$. Since $x \in I$, we have $z_{2}^{2} x=0$, so that $x=z_{1}^{2} x$. Hence, $\iota(\mathfrak{c})$ lies in $J\left(z_{1}\right)_{+}$, which is a natural cone in $Z^{\prime \prime}$. It follows that $\iota(\mathfrak{c})$ lies in the natural cone $\mathfrak{d}_{z_{1}}$ (see Lemma 4.15 (2) in [6]), which in turn is 
contained in $\mathfrak{d}_{z}=\mathfrak{e}$. Hence $\mathfrak{e}=\mathfrak{d}_{z_{1}}$. Since $\theta^{\prime \prime}$ takes squares of selfadjoint elements in $J\left(z_{1}\right)$ to squares of selfadjoints in $J(u)$, we have $\theta(\mathfrak{e}) \subset \mathfrak{d}$. Since $\theta$ takes natural cones to natural cones (by a variant of [5, Proposition 3.5]), by the definition of $\mathfrak{d}$ we must have $\theta(\mathfrak{e})=\mathfrak{d}$.

The ordered noncommutative Shilov boundary is particularly nice in the case that $X$ has a spanning cone: one may replace the cone $\mathfrak{e}$ defined in Theorem 2.2 by the entire positive cone of $Z$.

Theorem 2.3. Suppose that $X$ is an ordered operator space with a densely spanning cone, and that $i: X \rightarrow B$ is a positive complete isometry from $X$ into a $C^{*}$-algebra. Then the TRO A generated by $i(X)$ is a $C^{*}$-subalgebra of $B$ (and equals the $C^{*}$ algebra generated by $i(X))$. Moreover, the ordered ternary envelope of $X$ is a $C^{*}$ algebra, which we write as $\left(C_{e}^{*}(X), j\right)$. This $C^{*}$-algebra $C_{e}^{*}(X)$ has the following universal property: for $i: X \rightarrow A$ as above, there exists a (completely positive) surjective $*$-homomorphism $\theta: A \rightarrow C_{e}^{*}(X)$ satifying $\theta \circ i=j$.

Proof. This may be proved just as in [5, Corollary 5.4]. We include a quick direct proof. Simple algebra shows that $A^{2}$ and $A \cap A^{2}$ are $\mathrm{C}^{*}$-subalgebras of $B$. Also, if $x \in A \cap B_{+}$then $x^{2} \in\left(A^{2}\right)_{+}$, so that $x \in A^{2}$ (since square roots in a $\mathrm{C}^{*}$ algebra remain in the algebra). Thus $x \in A \cap A^{2}$. Hence $i\left(X_{+}\right) \subset A \cap A^{2}$, so that $i(X) \subset A \cap A^{2}$. This implies that $A \subset A \cap A^{2}$, which forces $A$ to be a $C^{*}$-subalgebra of $B$. The second statement is now fairly obvious.

By the first lines of the proof of Theorem 2.1, the ternary envelope of $X$ may be chosen to be a quotient of $A$ by a certain subspace. This subspace is a two-sided ideal if $A$ is a $C^{*}$-algebra, and so the quotient is a $C^{*}$-algebra which we write as $C$ below. Moreover, the canonical map $j: X \rightarrow C_{e}^{*}(X)$ is clearly also completely positive, being a composition of $i$ and the quotient $*$-homomorphism.

Given another map $\kappa: X \rightarrow B^{\prime}$ with the same properties as $i$ above, the TRO $D$ generated by $i(X)$ is a $\mathrm{C}^{*}$-algebra, by the above. By the universal property of the ternary envelope, there exists a ternary morphism $\pi: D \rightarrow C$ such that $\pi \circ \kappa=j$. It is easy algebra to check that $\pi$ is $*$-linear. Moreover, for example by the first part of the proof of [4, Corollary 8.3.5], the ternary morphism $\pi$ induces a $*$-homomorphism $\theta: D^{*} D=A \rightarrow C^{*} C=C$ defined by $\theta\left(d^{*} b\right)=\pi(d)^{*} \pi(b)$. If $x \in X_{+}$then $\kappa(x) \geq 0$, so that $\theta(\kappa(x)) \geq 0$. But also $j(x)=\pi(\kappa(x)) \geq 0$. Since $\theta(\kappa(x))^{*} \theta(\kappa(x))=\theta\left(\kappa(x)^{*} \kappa(x)\right)=\pi(\kappa(x))^{*} \pi(\kappa(x))$, it follows from the uniqueness of square roots that $\pi(\kappa(x))=\theta(\kappa(x))$. Since $\kappa\left(X_{+}\right)$generates $D$ as a TRO, this forces $\pi=\theta$.

Finally, we show that there is only one natural cone in $C_{e}^{*}(X)$ containing $X_{+}$. Any such cone may be viewed as the cone of a $*$-subTRO $W$ of a $C^{*}$-algebra, for which there is a ternary $*$-isomorphism $\rho: C \rightarrow W$ with $\kappa=\rho_{\mid X}$ positive. Applying the previous paragraph to $\kappa$, easily shows that $W$ is a $C^{*}$-algebra and $\rho^{-1}$ is a $*$-isomorphism, and hence an order isomorphism.

Indeed most of the other results in [5, Section 5] carry over to our setting.

We call $C_{e}^{*}(X)$ in Theorem 2.3, the $C^{*}$-envelope of $X$. If $X$ is a unital operator system then this coincides with the usual $\mathrm{C}^{*}$-envelope of Arveson and Hamana (see [1, 8] or [4, Section 4.3]).

Remarks. 1) If $X$ is as in Theorem 2.3, then the universal property there easily implies that there is a largest ordered operator space cone on $X$ containing the 
given cone $\mathfrak{c}=X_{+}$, namely $X \cap C_{e}^{*}(X)_{+}$. Thus the embedding of $X$ in $C_{e}^{*}(X)$ is a complete order embedding iff there is no strictly larger ordered operator space cone on $X$ containing $\mathfrak{c}$.

2) From the universal property it is easy to see that $C_{e}^{*}(X)$ is also an ordered ternary envelope of $X$ in the sense of [5].

We say that $X$ in Theorem 2.3 is e.n.v. if $C_{e}^{*}(X)$ is unital. This property only depends on the operator space structure of $X$ (not the order) [2]. In [2, Section 4], it is shown that if $X$ is a 'minimal operator space' (that is, $X$ is completely isometric to a subspace of a $C(K)$ space), then $X$ is e.n.v. iff 0 is not a weak* limit of extreme points of Ball $\left(X^{\prime}\right)$. Typically, spaces will not be e.n.v..

For the following result, we recall that the classical Shilov boundary is usually shown to exist for function spaces which contain constant functions. A space not containing constant functions may not have a Shilov boundary in the usual sense. We prove that this boundary does exist in a case we have not seen discussed in the classical literature:

Corollary 2.4. Suppose that $X$ is a closed selfadjoint subspace of $C_{0}(K)$, for a locally compact Hausdorff $K$, and suppose that the cone $X \cap C_{0}(K)_{+}$densely spans $X$. Then the Shilov boundary of $X$ exists as a topological space in the following sense: there exists a locally compact topological space $\partial X$, and a positive linear isometry $j: X \rightarrow C_{0}(\partial X)$ such that $j(X)$ separates points of $\partial X$ and does not vanish identically at any point, and such that for any other locally compact topological space $\Omega$ and positive linear isometry $i: X \rightarrow C_{0}(\Omega)$ such that $i(X)$ strongly separates points and does not vanish identically at any point, there exists a homeomorphism $\tau$ from $\partial X$ onto a subset of $\Omega$, such that $i(x) \circ \tau=j(x)$ for all $x \in X$. Also, $\partial X$ is compact iff $X$ is e.n.v..

Proof. The TRO inside $C_{0}(K)$ generated by $X$ is a $\mathrm{C}^{*}$-algebra $A$ by Theorem 2.3 , and it is commutative. By the universal property in Theorem 2.3, there is a *homomorphism from $A$ onto $C_{e}^{*}(X)$. Thus $C_{e}^{*}(X)$ is a commutative $\mathrm{C}^{*}$-algebra, so that $C_{e}^{*}(X)=C_{0}(\partial X)$ for a locally compact topological space $\partial X$, which will be compact if $X$ is e.n.v.. Clearly the copy of $X$ separates points of $\partial X$ and does not vanish identically at any point. Given any $(\Omega, i)$ as stated, then $\left(C_{0}(\Omega), i\right)$ is a ternary $*$-extension of $X$, so that by Theorem 2.3 there is a surjective *homomorphism $\pi: C_{0}(\Omega) \rightarrow C_{0}(\partial X)$ as in that Corollary, with $\pi \circ i=j$. By the well known dualities between categories of topological spaces and algebras of continuous functions, $\pi$ induces a homeomorphic embedding $\tau: \partial X \rightarrow \Omega$ such that $i(x) \circ \tau=j(x)$.

The $\mathrm{C}^{*}$-envelope in Theorem 2.3 and the Shilov boundary in the last result, will have (ordered versions of) almost all of the usual familiar properties of the noncommutative and classical Shilov boundaries (see e.g. [4, 9] for some of these). Thus it should be a useful tool. For example, it gives one a good handle on the completely positive surjective complete isometries $T: X \rightarrow Y$ between ordered operator spaces which are densely spanned by their positive cones. Indeed, such maps extend uniquely to $*$-isomorphisms between the $C^{*}$-envelopes, where in certain cases (such as the finite dimensional case considered at the end of the paper) they can be classified. To see this, note that the universal property in the Theorem 2.3. applied to $j^{\prime} \circ T$, gives a $*$-homomorphism $\pi: C_{e}^{*}(X) \rightarrow C_{e}^{*}(Y)$ such that $\pi \circ j=j^{\prime} \circ T$, where $j^{\prime}: Y \rightarrow C_{e}^{*}(Y)$ is the canonical map. On the other hand, 
by basic properties of the noncommutative Shilov boundary of any operator space, there is a ternary isomorphism $\theta: C_{e}^{*}(X) \rightarrow C_{e}^{*}(Y)$ such that $\pi \circ j=j^{\prime} \circ T$. So $\pi=\theta$ on $j(X)$, and hence on all of $C_{e}^{*}(X)$. So $\pi$ is bijective, and so is a $*$-isomorphism, and the uniqueness is obvious.

\section{UNITIZATIONS}

A natural way to attempt to understand nonunital ordered operator spaces is to 'unitize' them; that is to embed them as a codimension one subspace of a unital operator system (in the usual sense of e.g. 7]). This was first done in [16. That paper assigns, to any 'matrix ordered operator space' $X$, a unitization $X^{+}$, which is an operator system. Note $X^{+}$is spanned by $X$ and 1 , and the embedding $i: X \rightarrow X^{+}$is a completely contractive complete order embedding. One cannot hope that $i$ be isometric too, in general. To see this, suppose that $i$ was isometric in the case that $X=A^{\prime}$ is the dual of a nontrivial $\mathrm{C}^{*}$-algebra $A$, with its usual cone (which is a 'matrix ordered operator space'). Since $X^{+}$is an operator system, and hence may be viewed as a subspace of $B(H)$ containing $I_{H}$, we have contradicted Proposition 1.1. (We remark in passing that the same argument shows that the main result in [10] is not correct as stated; it has been corrected in [11.) It is easy to see from e.g. [16. Corollary 4.11], that $i$ is a complete isometry iff $X$ is a completely isometrically complete order embeddable in a $\mathrm{C}^{*}$-algebra. We mention, in passing, that such spaces were characterized in [16], as the spaces whose 'extended numerical radius' norms coincide with the given matrix norms.

It is shown in [16. Lemma 4.9 (c)] that $X^{+}$has the following universal property: for any completely contractive completely positive map $T$ from $X$ into a (unital) operator system $Y$, the extension $x+\lambda 1 \mapsto T(x)+\lambda 1$, from $X^{+}$into $Y$ is completely positive. Since the latter map is unital, it is also completely contractive. From this, it follows that $X^{+}$possesses the smallest cone a unitization of $X$ may have. To see this, take $Y$ in the universal property above to be any other unitization of $X$.

In contrast, it may well be useful sometimes to have a unitization with a 'biggest' positive cone. We will show that such a 'big unitization' exists if $X_{+}$densely spans $X$. In fact, after playing with examples, one sees that sometimes it is easier to describe this 'big unitization' explicitly than $X^{+}$(see e.g. the example at the end of the paper, or Corollary 3.5). For ordered operator spaces, we will define a unitization $X^{1}$ via the results in Section 2. Namely, if $X$ is an ordered operator space, let $W=\mathcal{T}^{o}(X)$ be its ordered ternary envelope. Since the positive cone of this is 'natural' in the sense of 6, by the results of that paper we may consider $W$ as a $*$-TRO inside a $\mathrm{C}^{*}$-algebra, with the inherited cone. Then $B=W+W^{2}$ is a $\mathrm{C}^{*}$-algebra (see [6]), and we set $X^{1}$ be the span of the image of $X$ and the identity of the $\mathrm{C}^{*}$-algebra unitization $B^{1}$ of $B$. There is a choice to make here if $B$ is already unital: in this paper we take $B^{1}=B$ in this case. Note that this choice has the consequence that $X^{1}=X$ if $X$ is already a unital operator system.

Theorem 3.1. Suppose that $X$ is an ordered operator space with a densely spanning cone (resp. $X$ is an ordered operator space), that $H$ is a Hilbert space, and that $i: X \rightarrow B(H)$ is a $*$-linear complete isometry which is completely positive (resp. completely positive and such that there is no smaller natural cone than $A \cap B(H)_{+}$ in the TRO A generated by $i(X)$ containing the image of the cone of $X)$. Then there is a completely positive unital map from $i(X)+\mathbb{C} I_{H} \rightarrow X^{1}$ extending the canonical map $i(X) \rightarrow X$. 
Proof. Let $\theta$ be the canonical ternary *-morphism from the TRO $A$ generated by $i(X)$ to $\mathcal{T}^{\circ}(X)$ (see Theorem 2.2 and Theorem 2.3). If $X$ has a densely spanning cone, then by Theorem 2.3. $A$ is a $\mathrm{C}^{*}$-algebra, and $\theta$ is a $*$-homomorphism, and hence extends to a $*$-homomorphism $\pi$ from a unitization of $A$, which we may take to be the span of $A$ and $I_{H}$, into a unitization of the $\mathrm{C}^{*}$-algebra $\mathcal{T}^{o}(X)$. We then restrict $\pi$ to the span of $i(X)$ and $I_{H}$.

In the 'respectively case', $\theta$ is positive by Theorem 2.2, and hence is positive as a map into the $\mathrm{C}^{*}$-algebra $B=W+W^{2}$ discussed above. By [6, Corollary 4.3] we can extend $\theta$ to a completely positive unital $*$-homomorphism from a unitization of $Z+Z^{2}$ into the unitization of $W+W^{2}$. Here $Z$ is the TRO generated by $i(X)$. Finally, restrict to the span of $i(X)$ and $I$ as before.

Corollary 3.2. If $X$ is an ordered operator space with a densely spanning cone, then $C_{e}^{*}\left(X^{1}\right)=C_{e}^{*}(X)^{1}$.

Proof. We assume that $C_{e}^{*}(X)$ is nonunital (the other case is similar but easier). By a basic property of the $\mathrm{C}^{*}$-envelope (see e.g. 8, 4]), it suffices to show that $J=0$ if $J$ is an ideal in $C_{e}^{*}(X)^{1}$ such that the canonical map $d: X^{1} \rightarrow C_{e}^{*}(X)^{1} / J$ is a complete isometry. Let $I=J \cap C_{e}^{*}(X)$, then the canonical map $C_{e}^{*}(X) \rightarrow C_{e}^{*}(X)^{1} / J$ factoring through $C_{e}^{*}(X)^{1}$, has kernel $I$. Hence $d$ restricted to $X$ may be viewed as a complete isometry into $C_{e}^{*}(X) / I$. By a basic property of the ternary envelope (see [9] or [4, 8.3.10]), we have $I=0$. Since $C_{e}^{*}(X)$ is an essential ideal in $C_{e}^{*}(X)^{1}$, we deduce that $J=0$. Thus $C_{e}^{*}\left(X^{1}\right)=C_{e}^{*}(X)^{1}$.

In this paragraph, let $X$ be an ' $L$-matricial Riesz normed space' in the sense of [15, 10, 11]. This is a subclass of the 'matrix ordered operator spaces' from [16] (to see that $X$ satisfies the first condition in Definition 3.3 of [16, use the formula in 10. Definition 2.3] and pre- and post-multiply the matrix there by the permutation matrix $U$ that switches around the four corners. The second condition is explicitly noted in [10]). In [10, 11] a unitization for such spaces $X$ is introduced, which we shall write as $\tilde{X}$. We show next that this unitization coincides with the one in [16. Thus one can apply results in [10, 11 to the setting of [16, and vice versa. For example, it follows from [11] that there is a nice 'internal' description the cone on $X^{+}$in the case that $X$ is ' $L^{\infty}$-matricial Riesz normed': namely, if $v \in M_{n}(X)$ and $A \in M_{n}$ are selfadjoint, then $(v, A)=\left[v_{i j}+a_{i j} 1\right]$ is in the positive cone of $M_{n}\left(X^{+}\right)$iff $A \geq 0$ and for any $\epsilon>0$ there is an $u \in M_{n}(X)_{+}$with $\|u\|<1$ so that $v+(A+\epsilon)^{1 / 2} u(A+\epsilon)^{1 / 2} \geq 0$ (see [10]). The first author thanks A. K. Karn for sending him a version of [11, which contains another variant of this formula that is not hard to see is equivalent to the one above (to see the nontrivial direction, note that if $\theta$ and $u$ are as in the definition of the cone in [11, put

$$
\hat{u}=(A+\epsilon 1)^{-1 / 2}(A+\theta)^{1 / 2} u(A+\theta)^{1 / 2}(A+\epsilon 1)^{-1 / 2}
$$

Since $A+\theta \leq A+\epsilon 1$ we have

$$
\left\|(A+\epsilon 1)^{-1 / 2}(A+\theta)^{1 / 2}\right\|^{2}=\left\|(A+\epsilon 1)^{-1 / 2}(A+\theta)(A+\epsilon 1)^{-1 / 2}\right\| \leq 1 .
$$

Thus $\|\hat{u}\|<1$, and $v+(A+\epsilon 1)^{1 / 2} \hat{u}(A+\epsilon 1)^{1 / 2}=v+(A+\theta)^{1 / 2} u(A+\theta)^{1 / 2} \geq 0$.)

Proposition 3.3. Whenever $X$ is as in the last paragraph, then $X^{+}=\tilde{X}$, completely positively and completely isometrically via the identity map. 
Proof. Consider the inclusion $X \rightarrow \tilde{X}$. By the property of $X^{+}$mentioned at the start of this section, this extends to a completely positive unital map $X^{+} \rightarrow \tilde{X}$. It therefore remains to show that every positive element $(v, A)$ in $M_{n}(\tilde{X})$ is positive in $M_{n}\left(X^{+}\right)$. Let $\epsilon>0$ be given, and let $u$ be as above, with $v+(A+\epsilon)^{1 / 2} u(A+\epsilon)^{1 / 2} \geq$ 0 . Then, for any positive contractive $\phi \in M_{n}(X)^{\prime}$,

$$
\phi\left((A+\epsilon)^{-1 / 2} v(A+\epsilon)^{-1 / 2}\right) \geq \phi(-u) \geq-1 .
$$

This is precisely the definition from [16] of the matrix cones of $X^{+}$. That is, by definition, $(v, A) \geq 0$ in $M_{n}\left(X^{+}\right)$.

As we see next, there seem to be interesting classes of ordered operator spaces $X$ for which $X^{+}=X^{1}$. Note that the latter condition implies, first, that $X \subset X^{+}$ completely isometrically. A unitization $\tilde{X}$ of $X$ will be called a completely isometric unitization if the canonical map $X \rightarrow \tilde{X}$ is completely isometric. Second, if $X^{+}=$ $X^{1}$ and if $X$ is densely spanned by $X_{+}$, then all completely isometric unitizations of $X$ coincide. This is because $X^{+}$is the smallest unitization, and Theorem 3.1]implies that $X^{1}$ has the biggest positive cone of any completely isometric unitization.

For example, we now study ordered operator spaces $X$ with the property that $X^{\prime \prime}$, with its induced positive cones (the canonical ones from e.g. [15], which are easy to see are just the weak* closure of $\left.M_{n}(X)_{+}\right)$, is a unital operator system (that is, it has an order unit making its cones Archimidean 7]). We sometimes write 1 for the identity $1_{X^{\prime \prime}}$ of $X^{\prime \prime}$. We will show that, under reasonable conditions, $X^{+}=X^{1}=\operatorname{Span}\left\{X, 1_{X^{\prime \prime}}\right\}$, the span in $X^{\prime \prime}$. In [10] a condition on 'approximate matrix order unit spaces' is given which characterizes when $\tilde{X}=\operatorname{Span}\left\{X, 1_{X^{\prime \prime}}\right\}$. We give a variant of this result, and include a short proof.

Lemma 3.4. (Cf. [10, Theorem 5.3]) Suppose that $X$ is an ordered operator space, and that $X^{\prime \prime}$ is a unital operator system as above. Then $X^{+}=\operatorname{Span}\left\{X, 1_{X^{\prime \prime}}\right\}$ iff there is no $v \in X$ dominating $1_{X^{\prime \prime}}$, and iff the distance $d(X, 1)$ of $1_{X^{\prime \prime}}$ from the copy of $X$ in $X^{\prime \prime}$ is 1 .

Proof. In this case, by the remark at the end of the first paragraph of this section, $X \subset X^{+}$completely isometrically. By the universal property of this construction, stated at the start of this section, the canonical map $i: X^{+} \rightarrow\left(X^{\prime \prime}\right)^{+}$is unital and completely positive. On the other hand, by definition of this unitization (see 16. Definition 4.7]), if $(v, A) \in M_{n}(X \oplus \mathbb{C})$ is in $M_{n}\left(\left(X^{\prime \prime}\right)^{+}\right)$then it is easy to see that it is in $M_{n}\left(X^{+}\right)$. Consequently $i$ is a complete order embedding. Thus $X^{+}$is easy to describe explicitly in terms of the simple unitization of the already unital operator system $X^{\prime \prime}$. Namely, the positive cone of $M_{n}\left(X^{+}\right)$consists of pairs $(v, A)$, with $v \in M_{n}(X)$ and $A \in\left(M_{n}\right)_{+}$, such that $v+A 1_{X^{\prime \prime}}$ is positive in $M_{n}\left(X^{\prime \prime}\right)$. It follows now from [16. Lemma 4.9(b)] that we can identify $X^{+}$with the span of $X$ and $1_{X^{\prime \prime}}$ in $X^{\prime \prime}$, iff whenever $v+A 1_{X^{\prime \prime}}$ is positive in $M_{n}\left(X^{\prime \prime}\right)$ then $A \geq 0$. As noted in [10], we can take $n=1$ in the latter condition. This, in turn, is clearly equivalent to there being no $v \in X$ dominating $1_{X^{\prime \prime}}$.

Suppose that $d(X, 1)<1$. Then there exists $x \in X$ with $\|x-1\|<1$. By the usual trick we may assume $x=x^{*}$. It follows by spectral theory that there is a $\lambda>0$ with $x \geq \lambda 1$, so that $x / \lambda \geq 1$. Conversely, if $d(X, 1)=1$, then by the Hahn-Banach theorem there exists a state $\varphi$ on $X^{\prime \prime}$ which annihilates the copy of $X$. If $v \in X$ with $v \geq 1_{X^{\prime \prime}}$, then $0=\varphi(v) \geq 1$, a contradiction. 
Theorem 3.5. If $X$ is an ordered operator space with $X^{\prime \prime}$ a unital operator system as above, then $X^{1}=\operatorname{Span}\left\{X, 1_{X^{\prime \prime}}\right\}$. In this case, $d(X, 1)=1$ iff all unitizations of $X$ coincide. In particular, the latter is true if $X$ is not e.n.v. (which is generally the case). This holds, for example, for any nonunital $C^{*}$-algebra.

Proof. Note that $X^{\prime \prime}$ is spanned by its positive elements, and it follows that $X$ is densely spanned by $X_{+}$. Hence the ordered ternary envelope of $X$ is a $\mathrm{C}^{*}$-algebra $C_{e}^{*}(X)$. By [3, Lemma 5.3] in conjunction with Theorem 2.3, we may view $C_{e}^{*}(X)$ as a $\mathrm{C}^{*}$-subalgebra $B$ of $C_{e}^{*}\left(X^{\prime \prime}\right)$. So if $X$ is not e.n.v., then we can identify $C_{e}^{*}(X)^{1}$ with the span of $B$ and $1_{X^{\prime \prime}}$. Thus in this case $X^{1}$ is the span of $X$ and $1_{X^{\prime \prime}}$ in $X^{\prime \prime}$, up to unital complete order isomorphism (and complete isometry). Similarly, if $X$ is e.n.v., with $1_{X^{\prime \prime}} \in C_{e}^{*}(X)$, then $X^{1}=\operatorname{Span}\left\{X, 1_{X^{\prime \prime}}\right\}$ by definition. Suppose that $X$ is e.n.v., but the identity $p$ of $C_{e}^{*}(X)$ is not $1_{X^{\prime \prime}}$. If $x \in X$ then $\left\|1_{X^{\prime \prime}}-x\right\|=\left\|1_{X^{\prime \prime}}-p x p\right\| \geq\left\|p^{\perp}\right\|=1$. Thus $d(X, 1)=1$. As in Lemma 3.4. there exists a state $\varphi$ on $X^{\prime \prime}$ annihilating the copy of $X$. Let $Y=\operatorname{Span}\{X, p\}$ and $Z=\operatorname{Span}\left\{X, 1_{X^{\prime \prime}}\right\}$, spans taken in $C_{e}^{*}(X)^{\prime \prime}$. The map $\Phi: z \mapsto p z p$ on $C_{e}^{*}\left(X^{\prime \prime}\right)$ restricts to a unital completely positive map $Z \rightarrow Y$. The canonical $*$-isomorphism $C_{e}^{*}(X)+\mathbb{C} 1_{X^{\prime \prime}} \cong C_{e}^{*}(X) \oplus^{\infty} \mathbb{C}$ taking $z=x+\lambda 1_{X^{\prime \prime}}$ to $(x+\lambda p, \lambda)$ for $x \in C_{e}^{*}(X), \lambda \in$ $\mathbb{C}$, restricts on $Z$ to the map $z \mapsto(\Phi(z), \varphi(z))$. Since $\varphi$ is completely contractive, this forces $\Phi$ to be completely isometric. Thus $\Phi$ is a completely isometric complete order isomorphism, so that $X^{1} \cong \operatorname{Span}\left\{X, 1_{X^{\prime \prime}}\right\}$.

If $X$ is not e.n.v. then the condition in Lemma 3.4 holds. This is clear if $X$ is a $\mathrm{C}^{*}$-algebra, and the general case reduces to this one as follows. In this case, we can identify $v+\lambda 1_{X^{\prime \prime}}$, as in the last paragraph, with $v+\lambda 1$ in $C_{e}^{*}(A)^{1}$. The condition in Lemma 3.4 now follows by the $\mathrm{C}^{*}$-algebra case.

The rest follows easily from the above proof, together with remarks and facts above the theorem.

Remark. If $X^{\prime \prime}$ is a unital operator system, as in the discussion above, then it is easy to argue that $X$ is 'maximally ordered': i.e. there is no larger ordered operator space cone on $X$. We leave the details to the interested reader.

Example. We illustrate the two main concepts of our paper in the very simple case of a selfadjoint subspace $X$ of matrices in $M_{n}$, by recalling for the general reader the usual recipe to compute the ordered noncommutative Shilov boundary; and this gives the unitization $X^{1}$ explicitly. In practice, for example given a concrete subspace of $M_{5}$ say, it is usually easy to implement this recipe. For simplicity in the discussion that follows, suppose that $X$ is spanned by $X \cap\left(M_{n}\right)_{+}$, which is equivalent to having a set of fewer than $n^{2}$ linearly independent positive matrices which span $X$ (the general case is only fractionally more complicated). Then by Theorem 2.3, the TRO generated in $M_{n}$ is also the $\mathrm{C}^{*}$-algebra $B$ generated by $X$ in $M_{n}$. However every finite dimensional $\mathrm{C}^{*}$-algebra 'is just' a direct sum of full matrix algebras $M_{k}$, for $k \leq n$. Thus, we may rewrite $X \subset B=B_{1} \oplus B_{2} \oplus \cdots \oplus B_{n}$, where each $B_{i}=M_{k}$, for some $k \leq n$. We call the $B_{i}$ 'blocks', and let $p_{i}$ be the minimal central projection in $B$ which 'is' just the identity of block $B_{i}$ (direct summed with several zeros). Some of these blocks may be unnecessary in the computation of norms of matrices with entries in $X$, and we call such a block 'loose'. More specifically, if $\|x\|=\left\|x\left(1-p_{i}\right)\right\|$ for all $x \in X$, and more generally for all $x \in M_{n}(X)$, then block $B_{i}$ is loose. If we find a loose block we remove it. For example, if block $B_{1}$ is loose, then we replace $B$ by $B^{\prime}=B_{2} \oplus \cdots \oplus B_{n}$, 
and replace $X$ by the subset of $B^{\prime}$ corresponding to $X\left(1-p_{1}\right)$. We then repeat the procedure. After less than $n$ steps, there will be no more loose blocks, and we will have arrived at the ternary *-envelope, which in this case is $C_{e}^{*}(X)$, and by construction it contains a completely isometric copy of $X$. The span of the identity of the latter $C^{*}$-algebra and the copy of $X$ inside this $C^{*}$-algebra, is $X^{1}$.

Acknowledgement. We thank A. K. Karn for a correction of a remark in an earlier version of this paper.

\section{REFERENCES}

[1] W. B. Arveson, Subalgebras of $C^{*}$-algebras, Acta Math. 123 (1969), 141-224.

[2] D. P. Blecher, The Shilov boundary of an operator space and the characterization theorems, J. Funct. Anal. 182 (2001), 280-343.

[3] D. P. Blecher, D. M. Hay, and M. Neal, Hereditary subalgebras of operator algebras, to appear J. Operator Theory.

[4] D. P. Blecher and C. Le Merdy, Operator algebras and their modules-an operator space approach, Oxford Univ. Press, Oxford (2004).

[5] D. P. Blecher and M. Neal, Open partial isometries and positivity in operator spaces, Preprint 2006.

[6] D. P. Blecher and W. Werner, Ordered $C^{*}$-modules, Proc. London Math. Soc. 92 (2006), 682-712.

[7] M.-D. Choi and E. G. Effros, Injectivity and operator spaces, J. Funct. Anal. 24 (1977), 156-209.

[8] M. Hamana, Injective envelopes of operator systems, Publ. R.I.M.S. Kyoto Univ. 15 (1979), $773-785$.

[9] M. Hamana, Triple envelopes and Silov boundaries of operator spaces, Math. J. Toyama University 22 (1999), 77-93.

[10] A. K. Karn, Adjoining an order unit to a matrix ordered space, Positivity 9 (2005), 207-223.

[11] A. K. Karn, Corrigedem to the paper "Adjoining an order unit to a matrix ordered space", Preprint, 2006.

[12] K. Kirkpatrick, The Shilov boundary and M-structure of operator spaces, Research Experiences for Undergraduates paper, Houston (2001).

[13] M. Neal, Inner ideals and facial structure of the quasi-state space of a JB-algebra, J. Funct. Anal. 173 (2000), 284-307.

[14] V. I. Paulsen, Completely bounded maps and operator algebras, Cambridge Studies in Advanced Math., 78, Cambridge University Press, Cambridge, 2002.

[15] W. J. Schreiner, Matrix regular operator spaces, J. Funct. Anal. 152 (1998), 136-175.

[16] W. Werner, Subspaces of $L(H)$ that are *-invariant, J. Funct. Anal. 193 (2002), 207-223.

[17] W. Werner, Multipliers on matrix ordered operator spaces and some K-groups, J. Funct. Anal. 206 (2004), 356-378.

Department of Mathematics, University of Houston, Houston, TX 77204-3008

E-mail address: dblecher@math.uh.edu

Department of Mathematics, Mit, 77 Massachusetts Avenue, Cambridge, Ma 021394307

E-mail address: kay@math.mit.edu

Department of Mathematics, Denison University, Granville, OH 43023

E-mail address: nealm@denison.edu

Mathematisches Institut, Einsteinstr. 62, D-48149 Münster, Germany

E-mail address: wwerner@math.uni-muenster.de 